\title{
MicroRNA-125b-5p suppresses Brucella abortus intracellular survival via control of A20 expression
}

\author{
Ning Liu', Lin Wang ${ }^{2}$, Changjiang Sun ${ }^{3}$, Li Yang $^{2}$, Wanchun Sun² and Qisheng Peng ${ }^{1,2^{*}}$
}

\begin{abstract}
Background: Brucella may establish chronic infection by regulating the expression of miRNAs. However, the role of miRNAs in modulating the intracellular growth of Brucella remains unclear.

Results: In this study, we show that Brucella. abortus infection leads to downregulation of miR-125b-5p in macrophages. We establish that miR-125b-5p targets A20, an inhibitor of the NF-kB activation. Additionally, expression of miR-125b-5p decreases A20 expression in B. abortus-infected macrophages and leads to NF-kB activation and increased production of TNFa. Furthermore, $B$. abortus survival is attenuated in the presence of miR-125b-5p.
\end{abstract}

Conclusions: These results uncover a role for miR-125b-5p in the regulation of B. abortus intracellular survival via the control of A20 expression.

Keywords: Brucella abortus, MiR-125, A20, Macrophage

\section{Background}

The genus Brucella is responsible for the worldwide Brucellosis, a disease of domestic and wild animals which is transmissible to humans [1]. The main symptoms include chills, fever, anorexia, and arthritis in humans and abortion in infected animals, which develops into a chronic infection if not treated [1].

Brucellae are intracellular pathogens that have the ability to survive and multiply inside professional phagocytic cells. The success of Brucella as an intracellular pathogen depends largely on its ability to avoid the activation of host macrophages upon infection [2, 3]. Brucella reprograms the host macrophage transcriptome by suppressing the expression of activation-related genes [4]. Noncoding RNAs, including microRNAs (miRNAs), may play an important role in this process through posttranscriptional regulation [5]. Diverse biological activities, including cell activation, are regulated by miRNAs

\footnotetext{
* Correspondence: Qishengpeng@yahoo.com

'Central Laboratory, The Second Hospital of Jilin University, Changchun 130041, China

${ }^{2}$ Key Laboratory for Zoonosis Research, Ministry of Education, Institute of Zoonosis, Jilin University, Changchun 130062, China

Full list of author information is available at the end of the article
}

[6]. In particular, miRNA expression changes have been described during macrophage infection with Brucella, suggesting that miRNAs are likely to influence the bacteria intracellular growth [7].

MiRNAs are single-stranded RNAs (approximately 22 nucleotides in length) that post-transcriptionally regulate gene and/or protein expression [8]. MiRNAs are species, cell, and context specific, and are dynamically regulated as a function of time in response to environmental triggers. A specific host miRNA response has been documented in the context of infection with intracellular bacteria, such as mycobacteria, Listeria monocytogenes, Francisella tularensis, and Salmonella enterica [9]. Nevertheless, to our knowledge, there has been no research to indicate that a specific host miRNA regulates Brucella intracellular survival.

The A20 protein, encoded by TNFAIP3, is an inhibitor of inflammation and is induced in many cell types by a wide range of stimuli [10]. It has been most intensively studied as an inhibitor of NF-kB activation and plays a key role in the inhibition of inflammation and innate immunity [11]. Recent studies have shown that miRNAs, such as miR-125a, miR-125b, and miR-873, can activate the NF-kB pathway by targeting the expression of A20 
[6, 12]. Additionally, in Mycobacterium tuberculosis-infected macrophages, downregulation of miR- let-7f was shown to enhance the expression of its target A20 [13], thereby attenuating inflammatory signaling and facilitating bacterial survival. Our previous study indicated that A20 promotes Brucella intracellular growth via inhibition of macrophage cell death and activation [14]. Considering that Brucella infection induces significant changes in miRNAs expression in macrophages [7], we have investigated whether miRNAs also participate in the regulation of Brucella growth by targeting A20 expression.

In this study, we report that the miR-125b-5p-mediated regulation of A20 (TNFAIP3) plays an important role in tuning the activation of $B$. abortus-infected macrophages. We demonstrate that the downregulation of miR-125b-5p during $B$. abortus infection enhances the expression of the A20 protein, thereby inhibiting NF-kB activation, and facilitating bacterial intracellular survival.

\section{Methods \\ Materials}

Anti- iNOS antibody was purchased from R\&D Systems (Minneapolis, MN, USA). Anti- ERK and calnexin were purchased from Cell Signaling Technology (Danvers, MA, USA). Anti-A20, $\beta$-actin, IkB $\alpha$ antibodies and Polybrene and puromycin were purchased from Santa Cruz Biotechnology (Dallas, Texas, USA). Enzyme-linked immunosorbent assay (ELISA) kit for TNFo measurement was purchased from eBioscience (San Diego, CA, USA).

\section{Mice}

C57BL/6 mice were obtained from commercial vendors and maintained under specific pathogen-free conditions in the animal facility of the Central Laboratory, The Second Hospital of Jilin University. The animal protocol was reviewed and approved by the Jilin University Institute Animal Care and Use Committee. The present investigations conform to the Guide for the Care and Use of laboratory Animals published by the US National Institutes of Health (NIH Publication No. 85-23, revised 1996).

\section{Brucella and macrophage cell line}

The Brucella abortus, strain 2308, used in this study, was originally obtained from Ding's laboratory at the China Institute of Veterinary Drug Control (Beijing, China) and was grown either in tryptic soy broth or on tryptic soy agar plates. The RAW264.7 macrophage and HEK293T cell lines were purchased from the American Type Culture Collection and cultured according to their instructions [14].

\section{Macrophage infection and survival assay}

Mice at 6-8 weeks of age were sacrificed by cervical dislocation. Femurs were obtained by using scissors to remove all muscle tissue from the bones. Bone marrow derived macrophages (BMDMs) were isolated from femurs by flushing with PBS using a 25-G needle as previously described [15]. Briefly, after 2 days, non-adherent BMDMs were collected and transferred at a density of $5 \times 10^{6}$ cells/plate and were cultured for an additional 2-5 days. BMDMs were maintained in $\alpha M E M$ supplemented with $10 \%$ endotoxin-free fetal bovine serum, 5 \% CMG (conditioned medium containing M-CSF), penicillin, streptomycin, and glutamine. The RAW264.7 cells and BMDMs were plated in 24-well plates in complete tissue culture media without antibiotics at a concentration of $2.0 \times 10^{5}$ cells per well and incubated overnight at $37{ }^{\circ} \mathrm{C}$ with $5 \% \mathrm{CO}_{2}$. The cells were infected with the B. abortus strain 2308 in triplicate wells at a multiplicity of infection (MOI) of 100:1 by centrifuging bacteria onto macrophages at $400 \mathrm{~g}$ for $10 \mathrm{~min}$ at $4{ }^{\circ} \mathrm{C}$. Following $15 \mathrm{~min}$ of incubation at $37{ }^{\circ} \mathrm{C}$ in a $5 \% \mathrm{CO}_{2}$ atmosphere, the cells were washed three times with

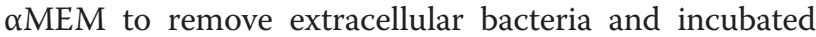
for an additional $60 \mathrm{~min}$ in medium supplemented with $50 \mu \mathrm{g} / \mathrm{ml}$ gentamicin to kill extracellular bacteria. To monitor $B$. abortus intracellular survival, infected cells were lysed with $0.1 \%$ Triton X-100 in phosphatebuffered saline (PBS), at certain time points, and serial dilutions of the lysates were rapidly plated onto tryptic soy agar plates to count the colony-forming units (CFUs) [16, 17].

\section{Production of lentivirus containing pre-miR-125b-5p or control Scr-miR}

Based on the analysis using the Targetscan software (http:// www.targetscan.org/), we found miR-125b-5p locus on chromosome 16: 77,644,273 to 77,648,343. The fragments of $\sim 400$ bp encompassing the miR-125b-5p sequences acted as the sequences of pre-miR-125b-5p. Next, the genomic DNA from the RAW264.7 cells was used as a template (the forward primer: ACGCGTAGATCTCACT GCTCTTGCAGATCT, the reverse primer: ACGCGTGC GGCCGCTTGGAACAGTGACTTGCT), and the premiR-125b-5p was PCR-amplified and cloned into the MSCV PIG (Puro IRES GFP) retrovirus vector at the BglII and XhoI cloning sites. The pMSCV PIG-Scr-miR vector was constructed by annealing of Scr-miR oligonucleotide sequences containing the BglII and XhoI cloning sites, ligation of the annealed oligonucleotides into the MSCV PIG vector. The Scr-miR sequence was ACGTCTATAC GCCCA. The generated plasmids were confirmed by DNA sequencing.

The lentivirus was produced by transiently transfecting HEK293T cells using the SuperFect transfection reagent (Qiagen, Redwood City, CA, USA) with three plasmid systems (pMSCV PIG-pre-125b-5p or pMSCV PIG-Scr-miR, psPAX2 and pMD2. G). The virus-containing supernatant 
was collected $72 \mathrm{~h}$ after transfection and filtered through a $0.45 \mathrm{~mm}$ filter (EMD Millipore, Billerica, MA, USA), and stored at $-80{ }^{\circ} \mathrm{C}$. Lentivirus titer on HEK293 cells was determined using the Adeno-X Rapid Titer kit (BD Biosciences Clontech, Palo Alto, CA, USA) according to the manufacturer's instructions.

\section{Establishment of the RAW264.7 stable cell expressing miR-125b-5p or control Scr-miR}

RAW264.7 cells were plated to $50 \%$ confluency in a 12well plate $24 \mathrm{~h}$ prior to viral infection. Next day, cells were infected with the miR-125b-5p or control Scr-miR lentivirus particles (the MOI was 10) overnight in antibiotics-free medium supplemented with $2 \%$ FBS and Polybrene at a final concentration of $5 \mu \mathrm{g} / \mathrm{ml}$. On the third day, the culture medium was replaced with $1 \mathrm{ml}$ of complete medium (without Polybrene), and the cells were incubated for a further 48. After a 5-day infection, cells that had stably incorporated the lentiviral construct were selected by survival in the presence of puromycin $(5 \mu \mathrm{g} / \mathrm{mL})$. Afterwards, the medium was replaced with fresh puromycin-containing medium every 3-4 days, until resistant colonies were identified. Finally, several colonies were picked, expanded and assayed by RT-PCR analysis for stable miR expression.

\section{RNA quantification}

RNA quantification was performed as previously described by Maegdefessel et al. [18]. Briefly, total RNA was isolated using a Trizol-based (Invitrogen, Carlsbad, CA, USA) RNA isolation protocol after RAW264.7 cells were infected with $B$. abortus at the indicated times. RNA was quantified by Nanodrop (Agilent Technologies, Santa Clara, CA, USA), and RNA and miRNA quality were evaluated using an Agilent 2100 Bioanalyzer (Agilent Technologies). First strand cDNA synthesis was performed for each RNA sample using the Superscript II RT Kit (Applied Biosystems, Waltham, MA, USA). Real time quantitative polymerase chain reaction was performed using TaKaRa PCR reagents (TaKaRa MiRNA qPCR Kit Ver. 2.0, TaKaRa Company, Kusatsu, Japan), the forward primer sequence for miR-125a-5p: AGTGTCCAATTTCCCAGAG, the forward primer sequence for miR-125b-5p: AGTGTTCAATCCCAGAG and the forward primer sequence for miR-351: GTCCGAGTTTCCC GAGGA. The reverse primers for miR-125a-5p, miR125b-5p and miR-351 were universal adaptor primers designed and provided by the TaKaRa Company. The relative amount of miRNA was standardized against U6 snRNA, and the fold change for miRNA was calculated using the 2-DD computed tomography method compared with relative control.

\section{Transient modulation of miR-125b-5p Level in RAW264.7} cells

Knockdown of miR-125b-5p expression in the RAW 264.7 cells was performed by transiently transfecting single-stranded anti-miR-125b-5p or control anti-miR oligonucleotides (Applied Biosystems), as previously described [6]. Briefly, $24 \mathrm{~h}$ before the experiment started, the culture media was changed and cells were maintained at $1 \times 10^{6}$ cells $/ \mathrm{mL}$. Immediately before electroporation, the cells were washed twice in ice-cold PBS and $5 \times 10^{6}$ cells $/ \mathrm{mL}$ were re-suspended in $500 \mu \mathrm{L}$ of serum-free medium with $100 \mathrm{nM}$ of the relevant oligo (GGAGUUGGAUUGCUGAAUU) in 4-mm cuvettes. Subsequently, electroporation was performed using a Bio-Rad Gene Pulser (Bio-Rad Laboratories, Hercules, CA, USA) [19]. Mature miR-125b-5p expression in RAW264.7 cells was determined by real-time RT-PCR analysis (TaKaRa MiRNA qPCR Kit Ver. 2.0). The primer for the miR-125b-5p assay is shown in the method section 2.6.

\section{Western blot}

Macrophages were lysed in ice-cold radioimmunoprecipitation assay (RIPA) buffer. The protein content was assayed by the BCA protein assay reagent (Pierce, Waltham, MA, USA). Twenty micrograms of protein were loaded into and size-separated by SDS-PAGE and then transferred to PVDF membrane. Afterwards, the membrane was incubated with a 1:1000 dilution of the primary antibody, followed by a 1:2000 dilution of horseradish peroxidase-conjugated secondary antibody. Protein bands were visualized by ECL (GE Healthcare, Chicago, Il, USA). The band densities were measured by densitometry (model GS-700, Imaging Densitometer; Bio-Rad), and the background was subtracted from the calculated area [20].

\section{Determination of NO production}

The supernatant of each cell sample was collected at certain times point after the $B$. abortus challenge. Nitric oxide (NO) content was measured by analyzing its stable end product, nitrite, using the Griess reagent (Sigma-Aldrich Company, Saint Louis, MO, USA) as previously described [21]. Data are expressed as micromoles of nitrite (mean \pm SEM).

\section{TNFa measurement}

RAW264.7 cells were infected with $B$. abortus for the indicated times. The supernatants were collected for analyzing the secretion of TNF $\alpha$ using an enzyme-linked immunosorbent assay (ELISA) kit (eBioscience, San Diego, CA, USA) according to the manufacturer's instructions [22]. 
Immunofluorescent confocal microscopy

Immunofluorescent confocal microscopy visualization was performed as described previously [23]. Briefly, RAW264.7 macrophages were seeded in 8- or 16-well Permanox chamber slides (Costar Hatfield, PA, USA) and infected with GFP-expressing B. abortus for the indicated times. Following infection, cells were fixed with $1 \%$ paraformaldehyde in PBS, permeabilized with $0.05 \%$ saponin in PBS for $30 \mathrm{~min}$ at room temperature. After being blocked with antibody 2.4G2 for $1 \mathrm{~h}$ at room temperature, the slides were incubated with DAPI and the primary antibody (calnexin antibody) overnight at $4{ }^{\circ} \mathrm{C}$. Subsequently, cells were washed with PBS and incubated for $1 \mathrm{~h}$ at room temperature with the fluorescein isothiocyanate (FITC)-conjugated secondary antibody (Alexa Fluor 568), as described in the Invitrogen protocol. Samples were visualized with a Zeiss LSM510 confocal microscope and the images were analyzed with the LSM confocal software [23].

\section{Statistical analysis}

Statistical analysis was performed using the SPSS10.0 software (IBM Corporation, Armonk, NY, USA). Data are expressed as mean \pm SEM. The statistical significance of the differences was evaluated by one-way ANOVA followed by the Student's $t$-test. $P<0.05$ was considered statistically significant [23].

\section{Results}

MiR-125b-5p is downregulated in B. abortus-infected macrophages

We previously reported that $B$. abortus infection enhanced the expression of A20 in macrophages, but the exact mechanism remains elusive. Considering that Brucella can establish chronic infection in part by regulating the expression of miRNAs [7], we hypothesized that the downregulation of a specific miRNA targeting A20 may be linked to the concomitant upregulation of A20 in $B$. abortus-infected macrophages. To investigate this hypothesis, we used TargetScan (http://www.targetscan.org/) to predict the intersection of target genes of miRNAs. Several miRNAs, including miR-351, miR125a-5p, miR-125b-5p, miR-6394 and miR-6367 are predicted to potentially target A20, as shown in Fig. 1a. Given that miR-6394 and miR-6367 were not detected in the microRNA expression profile of Brucella-infected RAW264.7 cells [7], miR-351, miR-125a-5p and miR$125 \mathrm{~b}-5 \mathrm{p}$ were chosen to test the biological relevance of

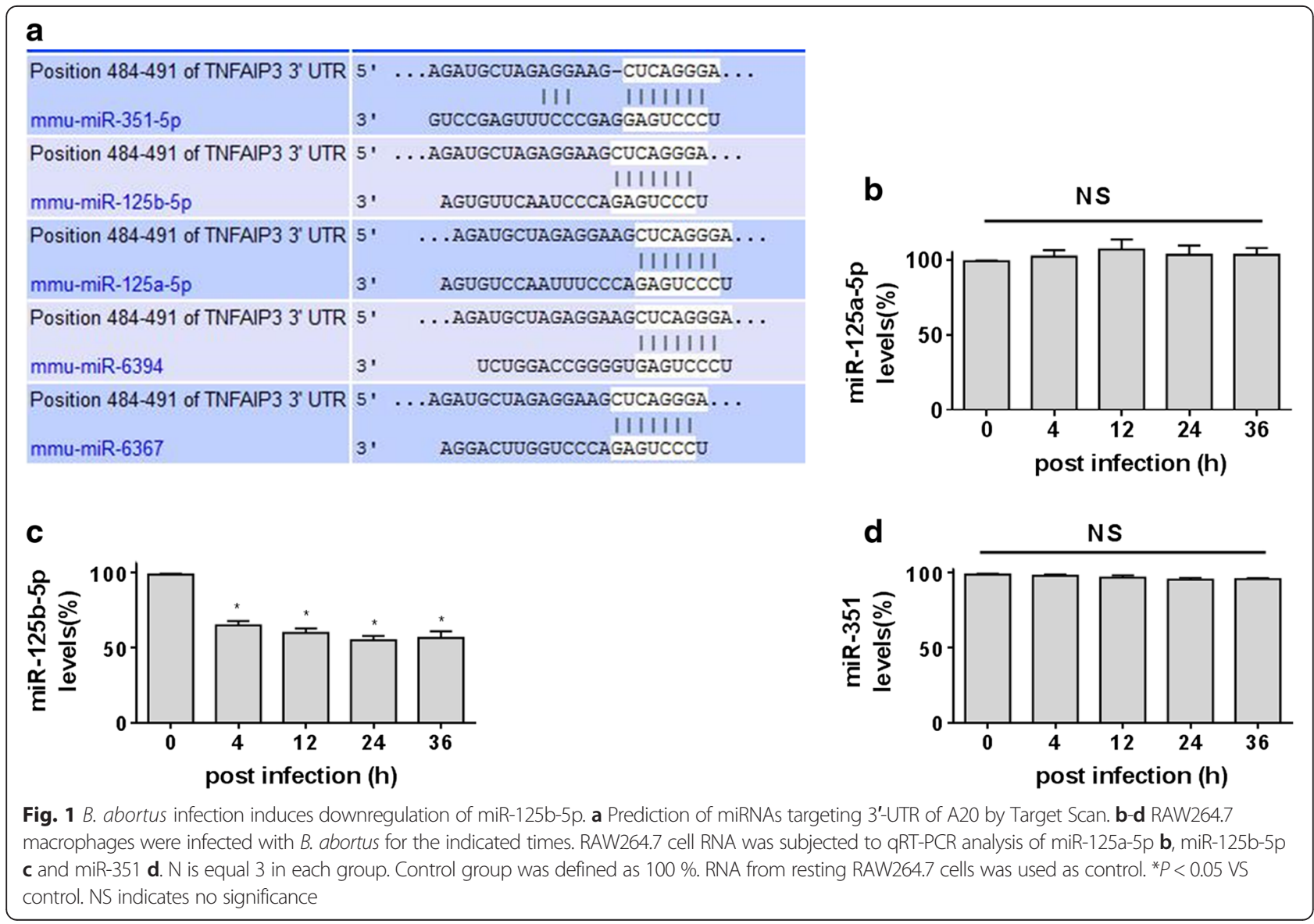


the downregulation of these miRNAs during infection by Brucella. RAW264.7 cells were infected with $B$. abortus at a MOI of 100 and the miRNA expression was quantified. The RNA was isolated from infected macrophages over time and the fold change for miRNAs was calculated using the 2-DD computed tomography method after the relative amount of miRNA was standardized against the U6 snRNA. The expression of miR-125b-5p, but not miR-351 and miR-125a-5p, was decreased at $4 \mathrm{~h}$ with peak suppression at $24 \mathrm{~h}$ of $B$. abortus infection, as shown in Fig. $1 \mathrm{~b}$ and $\mathrm{d}$. This result indicates that the miR-125b-5p is specifically suppressed during Brucella infection. Actually, previous work has found that miR-125b-5p directly bind to the A20 gene 3'-UTR [6]. Therefore, the miR-125b-5p is likely to participate in the upregulation of the expression of A20 during Brucella infection.

\section{A20 is a target of miR-125b-5p}

As A20 is not expressed in the resting RAW264.7 cells, it is hard to test whether the miR-125b-5p is associated with upregulation of the A20 protein. To address this problem, the RAW264.7 cells were primed with $10 \mathrm{ng} /$ $\mathrm{ml}$ of TNF $\alpha$ for $4 \mathrm{~h}$ to significantly increase the expression of A20 (Fig. 2a). In order to investigate whether A20 is a target of miR-125b-5p, RAW264.7 cells were infected with the lentivirus expressing pre-miR-125b-5p or with control scrambled microRNA (Scr-miR) and were treated with TNF $\alpha$ to induce the expression of
A20. RAW264.7 cells infected with scr-miR upregulated A20 in response to TNF $\alpha$, whereas pre-miR-125b-5p infected cells had a blunted response with significantly less A20 upregulation (Fig. 2b). To determine the functional consequences of the A20 regulation by miR-125b-5p, we evaluated NF-kB activation in response to the toll-like receptor ligands in cells expressing pre-miR-125b-5p [13]. RAW264.7 cells transfected with scr-miR or miR$125 \mathrm{~b}-5 \mathrm{p}$ were treated with $1000 \mathrm{ng} / \mathrm{ml} E$. coli LPS and NF- $k B$ activation determined as measured by the degradation of $\mathrm{IkB} \alpha$ [24]. $\mathrm{IkB} \alpha$ was degraded at 20 and $40 \mathrm{~min}$ in response to LPS in both the Scr-miR and miR-125b-5p transfected cells; however, IkB $\alpha$ remained low at $60 \mathrm{~min}$ in the miR-125b-5p transfected cells, indicating persistent NF-kB activation (Fig. 1c). Taken together, these data suggest that A20 is a target of miR125b-5p.

\section{B. abortus infection modulates the expression of A20 and NF-kB activity in a miR-125b-5p-dependent manner}

To test whether $B$. abortus infection induces downregulation of miR-125b-5p to regulate the expression of A20, we evaluated the expression of A20 in B. abortus infectedRAW264.7 cells. The results revealed that A20 was markedly upregulated after $4 \mathrm{~h}$ post infection and remained elevated for $24 \mathrm{~h}$, as shown in Fig. 3a. RAW264.7 cells were transduced with the lentivirus containing pre-miR$125 \mathrm{~b}-5 \mathrm{p}$ or Scr-miR prior to B. abortus infection. It was found that A20 expression was decreased significantly in

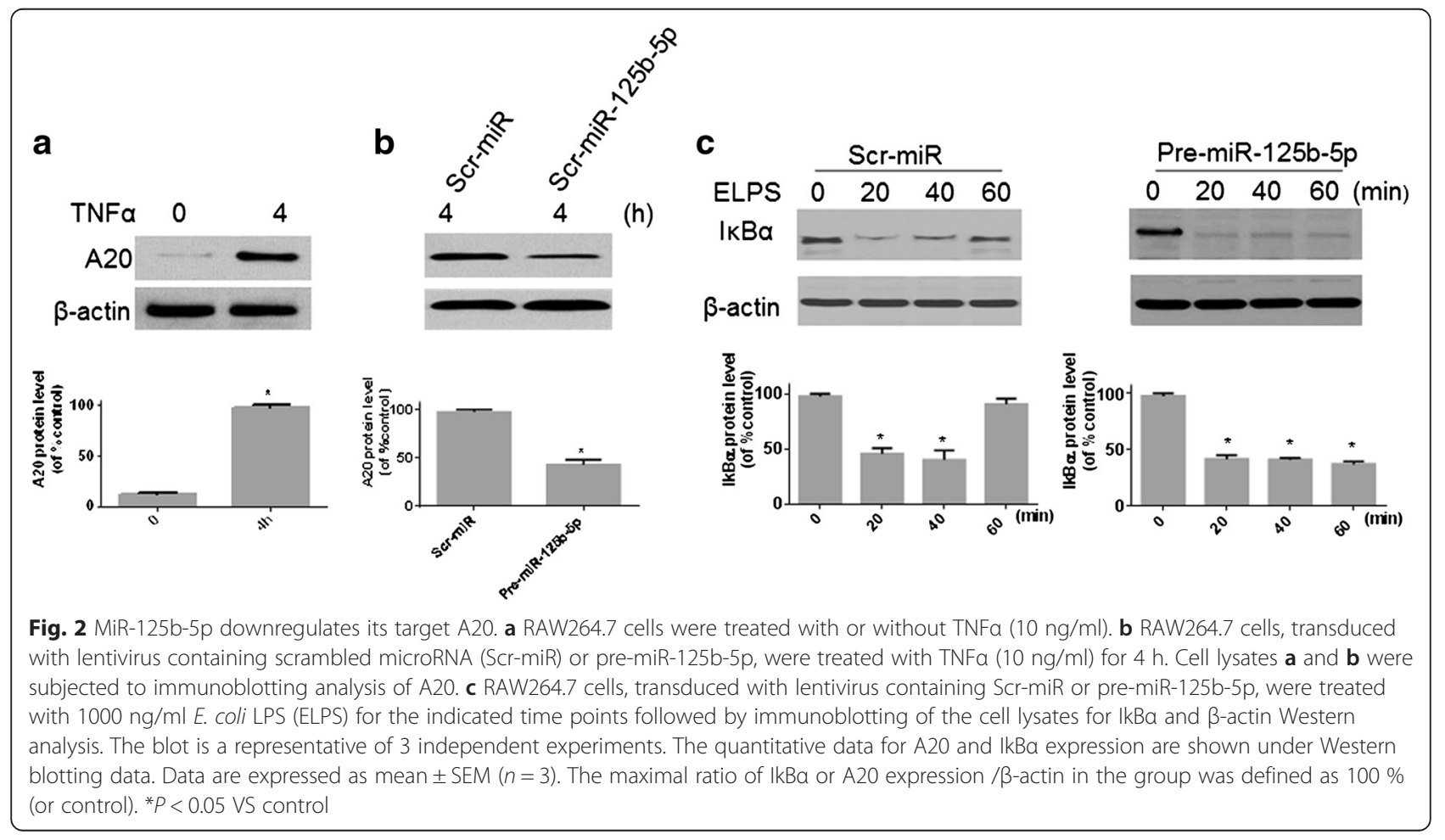


a

$\beta$-actin

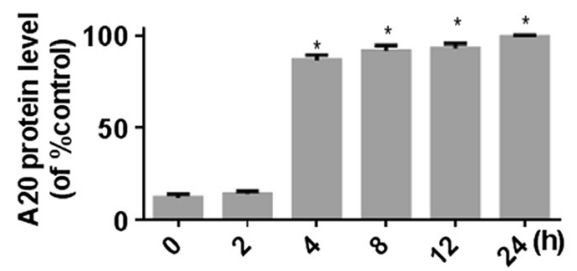

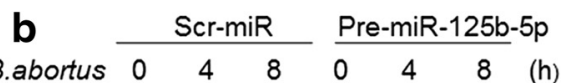

(h)

A20

$\beta$-actin
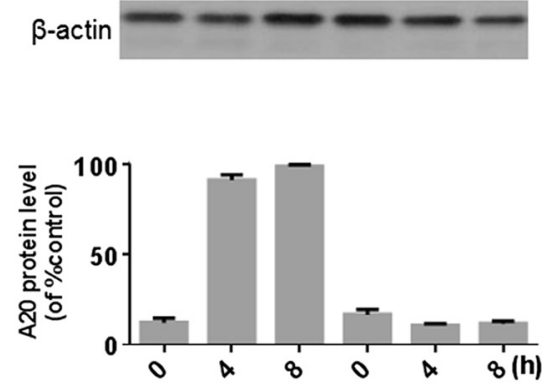
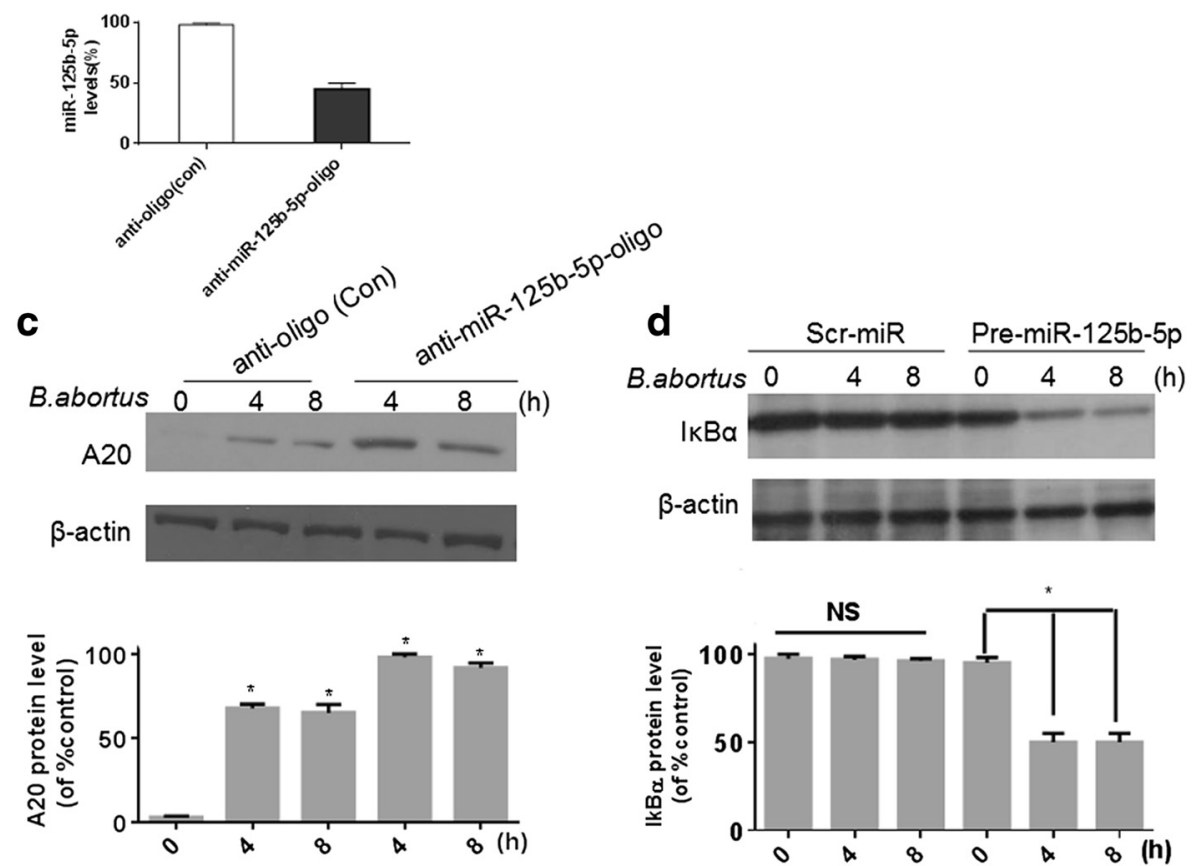

Fig. 3 B. abortus infection modulates the expression of A20 and IkBa in a miR-125b-5p-dependent manner. a RAW264.7 cells were infected with B. abortus for different periods of time. b RAW264.7 cells were transfected with either Scr-miR or Pre-miR-125b-5p and infected with B. abortus for 4 or $8 \mathrm{~h}$. Expression of A20 in $\mathbf{a}$ and $\mathbf{b}$ were determined by immunoblotting with A20 antibody. $\mathbf{c}$ RAW264.7 cells transfected with control anti-oligonucleotides (anti-oligo (Con)) or anti-miR-125b-5p oligonucleotides (anti- miR-125b-5p-oligo) were infected with B. abortus and their RNA was subjected to qRT-PCR analysis of miR-125b-5p (top panel). A20 expression in cell lysates was measured by immunoblotting with A20 antibody. d RAW264.7 cells transfected with Scr-miR or Pre-miR-125b-5p were infected with B. abortus for the indicated time periods, and IkBa expression was monitored in RAW264.7 cell lysates with IkBa antibody. The blot is representative of 3 independent experiments. Quantitative data for A20 and IkBa expression are shown under the Western blotting data. Data are expressed as mean $\pm \operatorname{SEM}(n=3)$. The maximal ratio of $1 \mathrm{kBa}$ or A20 expression / $\beta$-actin in the group was defined as $100 \%$ (or control). * $P<0.05$ VS control. NS indicates no significance

infected cells expressing pre-miR-125b-5p compared to cells expressing control Scr-miR (Fig. 3b). These results indicate that miR-125b-5p regulates A20 expression in $B$. abortus-infected macrophages. In order to further confirm the direct involvement of miR-125b-5p on the regulation of A20 expression, we also transfected cells with anti-miR- 125b-5p oligonucleotides to knock down the miR-125b-5p levels prior to infection with $B$. abortus (Fig. 3c, top panel). Following infection of the RAW264.7 cells, A20 levels were increased significantly compared to cells transfected with control anti-oligo (Fig. 3c, second panel), supporting the argument that miR-125b-5p is 
directly linked to the regulation of A20 expression in $B$. abortus-infected cells.

Since A20 is a feedback inhibitor of NF-kB signaling [24], the $B$. abortus infection-induced downregulation of miR-125b-5p should inhibit NF-kB activation. To test this notion, cells transfected with a pre-miR-125b-5p or Scr-miR were infected with $B$. abortus and analyzed. Specifically, NF-kB activation was measured by monitoring the degradation of $\mathrm{IkB} \alpha$. The results revealed that compared to cells transfected with control Scr-miR, IkB $\alpha$ was significantly degraded in RAW264.7 cells transfected with pre-miR-125b-5p indicating, as shown in Fig. 3d, that the miR-125b increased NF-kB activity. Taken together, these data demonstrate that B. abortus infection modulates the expression of A20 and NF-kB activity in a miR-125b-5p-dependent manner.

\section{B. abortus-induced downregulation of miR- $125 b-5 p$ is linked to $B$. abortus intracellular growth via regulating macrophage activation}

Having shown that $B$. abortus infection induces the suppression of miR-125b-5p and induction of A20 expression, we further investigated the role of miR-125b-5p in $B$. abortus infection. To ascertain the role of miR-125b$5 \mathrm{p}$ in bacterial intracellular survival, the $B$. abortus CFUs were counted in infected RAW264.7 cells after transfection with either Scr-miR or Pre-miR-125b-5p. The results indicate, as evident in Fig. 4a, that survival of $B$. abortus was significantly decreased in the presence of miR-125b-5p compared to Scr-miR. To assess the bacterial burden, confocal microscopy was also used to examine the intracellular bacteria colocalization with the endoplasmic reticulum (ER) marker calnexin at $36 \mathrm{~h}$ post infection.

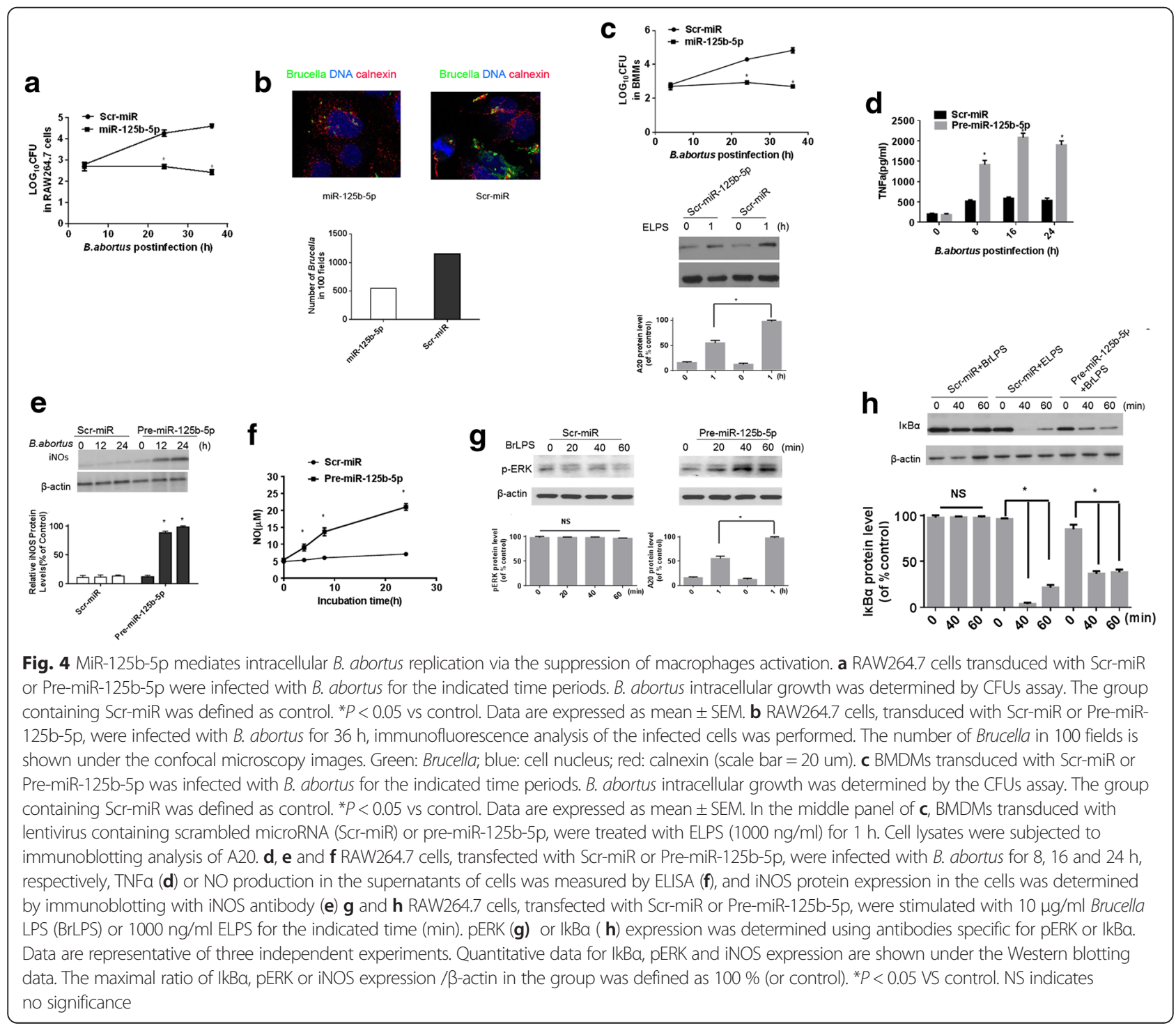


Consistent with the CFU assay, the number of B. abortus in the ER was markedly decreased in the RAW264.7 cells transfected with pre-miR-125b-5p (Fig. 4b). Additionally, BMDMs transfected with pre-miR-125b-5p were used to confirm the role of miR-125b-5p in mediating Brucella intracellular growth. Our data showed that the regulatory role of miR-125b-5p in BMDMs was similar to that observed in RAW264.7 cells (Fig. 4c). Collectively, our results suggest that the downregulation of miR-125b-5p facilitates the survival of $B$. abortus in macrophages.

We next examined the mechanism by which the downregulation of miR-125b-5p induced by $B$. abortus facilitates its intracellular replication. Since activated macrophages are the primary source for Brucella elimination in the infected host $[3,25,26]$, we hypothesized that miR-125b-5p mediates bacterial intracellular growth by regulating macrophages activation. Considering that the increase of TNF $\alpha$ production is the hallmark of activated macrophages [27], TNF $\alpha$ production was examined after RAW264.7 cells were infected with B. abortus. The results showed that TNF $\alpha$ was markedly increased in the presence of pre-miR-125b-5p compared to Scr$\mathrm{miR}$ in response to $B$. abortus infection, as shown in Fig. 4c. To further confirm that miR-125b-5p regulates macrophage activation after infection with Brucella, we also evaluated iNOS expression, NO production, and activation of ERK and NF-kB as additional markers of macrophages activation [3, 28, 29]. iNOS expression (Fig. 4d) and NO production (Fig. 4e) were prominently increased in the presence of pre-miR-125b-5p compared to Scr-miR after B. abortus infection indicating that there is increased macrophage activation.

Brucella LPS (BrLPS) is generally reported to be a poor inducer of inflammatory cytokines and macrophage activation $[1,30]$. To determine whether miR-125b-5p regulates ERK activation, RAW264.7 cells expressing Scr-miR or pre-miR-125b-5p were stimulated with BrLPS and ERK activation was examined. The results revealed that ERK was significantly enhanced in premiR-125b-5p cells compared to Scr-miR cells (Fig. 4f and g). Additionally, we also observed that stimulation of cells with BrLPS in the presence of pre-miR-125b-5p triggered an increase of NF-kB activity similar to that observed with the more potent E. coli LPS stimulation (Fig. 4g). Taken together, these data indicate that $B$. abortus-induced downregulation of miR-125b-5p mediates $B$. abortus intracellular growth via suppression of macrophages activation.

\section{Discussion}

Brucella has the ability to subvert the innate immune defense of host macrophages in order to establish infection within the macrophages. Brucella may establish Brucella-containing vacuole (BCV) for its intracellular growth by regulating the expression of miRNAs [7]. However, no studies have focused on how the utilization of the miRNAs during Brucella infection influences bacterial survival. Our data have revealed that the miR$125 \mathrm{~b}-5 \mathrm{p}$ is downregulated in B. abortus infection. Focusing on miR-125b-5p, we have demonstrated that its downregulation is associated with the concomitant upregulation of one of its target, namely A20, which mediates $B$. abortus intracellular replication via the regulation of NF-kB activity and macrophage activation.

Considering that NF-kB is a prominent regulator of macrophage activation [31], we focused our investigation on the putative miR-125b-5p target A20, which is an important regulator of NF-kB activation. The upregulation of A20 in B. abortus-infected macrophages was decreased in the presence of a miR-125b-5p and increased when cells were transfected with anti-miR-125b-5p, suggesting that the $B$. abortus-induced suppression of miR-125b-5p is linked to the enhanced expression of A20. At the same time, transfection of the pre-miR125b-5p was associated with increased NF-kB activity, suggesting that regulation of A20 by miR-125b-5p likely mediates NF-kB activity during $B$. abortus infection.

We have reported that A20 promotes B. abortus intracellular growth via inhibition of macrophage activation and their death. Our observations now add a further layer of complexity to this fine-tuning of macrophage activation by adding a miRNA-dependent mechanism of regulation of A20. Accordingly, we propose that the $B$. abortus-induced suppression of miR-125b-5p leads to increased A20 expression and concomitant reduction of macrophage activation via suppression of TNF $\alpha$, which is required for macrophage activation [27]. The observed suppression of nitrite production likely facilitates the survival of Brucella in macrophages [28]. This hypothesis is also supported by our data that transfection of the miR-125b-5p prior to infection is associated with ERK activation, another marker of macrophage activation, which is consistent with the findings in TREM-2 knockdown macrophages [2]. Our results demonstrate an unappreciated role of miR-125b-5p downregulation in favoring B. abortus intracellular survival.

In conclusion, this study provides reference points for further investigation into understanding the mechanism of Brucella intracellular survival mediated by miRNAs. The data in Brucella-infected macrophages support the notion that miRNA may play an important role in favor of Brucella survival. It is tempting to speculate that targeted intervention of essential miRNA could provide an attractive therapy for the management of Brucellosis.

\section{Conclusions}

The following conclusions can be drawn from our results: 1) MiR-125b-5p is downregulated in Brucella- 
infected macrophages; 2) Brucella infection suppresses NF-kB activity in a miR-125b-5p-dependent manner; 3) MiR-125b-5p inhibits Brucella -infected macrophage activation.

\section{Abbreviations}

$\mathrm{BCV}$, Brucella-containing vacuole; BMDMs, Bone marrow derived macrophages; BrLPS, Brucella LPS; CFUs, the colony-forming units; CMG, conditioned medium containing M-CSF; ELISA, Enzyme-linked immunosorbent assay; ER, endoplasmic reticulum; FITC, fluorescein isothiocyanate; miRNAs, microRNAs; $\mathrm{MOI}$, multiplicity of infection; RIPA, radioimmunoprecipitation assay; Scr-miR, scrambled microRNA

\section{Acknowledgments}

We thank Dr. Mary Beth Humphrey (Department of Medicine, University of Oklahoma Health Sciences Center, Oklahoma City, OK) for critically reading this manuscript.

\section{Funding}

This work was funded by National Natural Science Foundation of China (31372409) to QishengPeng. This work was also supported by Natural Science Foundation of China (81472030, 21175055), Jilin Province Science and Technology Department (20110739, 20150204001YY), Health and Family Planning Commission of Jilin Province (2015Z041), and Jilin University Bethune Project B (2012210) to Ning Liu.

\section{Availability of data and materials}

The data sets supporting the results of this article are included within the article and its additional file.

\section{Authors' contributions}

NL, LW, CJ and LY conceived of the study and carried out all experimental work and data analyses. NL and QS drafted the manuscript. WC was involved in critical revisions of the manuscript. All authors read and approved the final manuscript.

\section{Competing interests}

The authors declare that they have no competing interests.

\section{Consent for publication}

Not applicable.

\section{Ethics approval and consent to participate}

The animal protocol was reviewed and approved by the Jilin University Institute Animal Care and Use Committee.

\section{Author details}

${ }^{1}$ Central Laboratory, The Second Hospital of Jilin University, Changchun 130041, China. ${ }^{2}$ Key Laboratory for Zoonosis Research, Ministry of Education, Institute of Zoonosis, Jilin University, Changchun 130062, China. ${ }^{3}$ College of Veterinary Medicine, Jilin University, Changchun 130062, China.

\section{Received: 20 November 2015 Accepted: 27 July 2016}

\section{Published online: 29 July 2016}

\section{References}

1. Martirosyan A, Moreno E, Gorvel JP. An evolutionary strategy for a stealthy intracellular Brucella pathogen. Immunol Rev. 2011;240(1):211-34.

2. Wei P, Lu Q, Cui GM, Guan ZH, Yang L, Sun CJ, Sun WC, Peng QS. The role of TREM-2 in internalization and intracellular survival of Brucella abortus in murine macrophages. Vet Immunol Immunopathol. 2015; 163(3-4):194-201.

3. Barquero-Calvo E, Chaves-Olarte E, Weiss DS, Guzman-Verri C, Chacon-Diaz C, Rucavado A, Moriyon I, Moreno E. Brucella abortus uses a stealthy strategy to avoid activation of the innate immune system during the onset of infection. Plos One. 2007;2(7), e631

4. Wang F, Hu S, Liu W, Qiao Z, Gao Y, Bu Z. Deep-sequencing analysis of the mouse transcriptome response to infection with Brucella melitensis strains of differing virulence. Plos One. 2011;6(12), e28485.

5. Rajaram MVS, Ni B, Morris JD, Brooks MN, Carlson TK, Bakthavachalu B, Schoenberg DR, Torrelles JB, Schlesinger LS. Mycobacterium tuberculosis lipomannan blocks TNF biosynthesis by regulating macrophage MAPK- activated protein kinase 2 (MK2) and microRNA miR-125b. Proc Natl Acad Sci U S A. 2011:108(42):17408-13.

6. Kim SW, Ramasamy K, Bouamar H, Lin AP, Jiang DF, Aguiar RCT. MicroRNAs miR-125a and miR-125b constitutively activate the NF-kappa B pathway by targeting the tumor necrosis factor alpha-induced protein 3 (TNFAIP3, A20). Proc Natl Acad Sci U S A. 2012;109(20):7865-70.

7. Zheng K, Chen DS, Wu YQ, Xu XJ, Zhang H, Chen CF, Chen HC, Liu ZF. MicroRNA Expression Profile in RAW264.7 cells in Response to Brucella melitensis Infection. Int J Biol Sci. 2012;8(7):1013-22.

8. O'Connell RM, Rao DS, Baltimore D. microRNA regulation of inflammatory responses. Annu Rev Immunol. 2012;30:295-312.

9. Maudet C, Mano M, Eulalio A. MicroRNAs in the interaction between host and bacterial pathogens. Febs Letters. 2014;588(22):4140-7.

10. Beyaert R, Heyninck K, Van Huffel S. A20 and A20-binding proteins as cellular inhibitors of nuclear factor-kappa B-dependent gene expression and apoptosis. Biochem Pharmacol. 2000;60(8):1143-51.

11. Karin M, Greten FR. NF-kappaB: linking inflammation and immunity to cancer development and progression. Nat Rev Immunol. 2005:5(10):749-59.

12. Balkhi MY, Iwenofu OH, Bakkar N, Ladner KJ, Chandler DS, Houghton PJ, London CA, Kraybill W, Perrotti D, Croce CM, et al. miR-29 acts as a decoy in sarcomas to protect the tumor suppressor A20 mRNA from degradation by HuR. Sci Signal. 2013:6(286):ra63.

13. Kumar M, Sahu SK, Kumar R, Subuddhi A, Maji RK, Jana K, Gupta P, Raffetseder J, Lerm M, Ghosh Z, et al. MicroRNA let-7 Modulates the Immune Response to Mycobacterium tuberculosis Infection via Control of A20, an Inhibitor of the NF-kappa B Pathway. Cell Host Microbe. 2015;17(3):345-56.

14. Wei P, Cui G, Lu Q, Yang L, Guan Z, Sun W, Zhao Y, Wang S, Peng Q. A20 promotes Brucella intracellular growth via inhibition of macrophage cell death and activation. Vet Microbiol. 2015:175(1):50-7.

15. Humphrey MB, Daws MR, Spusta SC, Niemi EC, Torchia JA, Lanier LL, Seaman WE, Nakamura MC. TREM2, a DAP12-associated receptor, regulates osteoclast differentiation and function. J Bone Miner Res Off J Am Soc Bone Miner Res. 2006:21(2):237-45

16. Celli J, de Chastellier C, Franchini DM, Pizarro-Cerda J, Moreno E, Gorvel JP. Brucella evades macrophage killing via VirB-dependent sustained interactions with the endoplasmic reticulum. J Exp Med. 2003;198(4):545-56.

17. He Y, Reichow S, Ramamoorthy S, Ding X, Lathigra R, Craig JC, Sobral BW, Schurig GG, Sriranganathan N, Boyle SM. Brucella melitensis triggers timedependent modulation of apoptosis and down-regulation of mitochondrion-associated gene expression in mouse macrophages. Infect Immun. 2006;74(9):5035-46.

18. Maegdefessel L, Azuma J, Toh R, Merk DR, Deng A, Chin JT, Raaz U, Schoelmerich AM, Raiesdana A, Leeper NJ, et al. Inhibition of microRNA-29b reduces murine abdominal aortic aneurysm development. J Clin Investig. 2012;122(2):497-506

19. Rai D, Kim SW, McKeller MR, Dahia PL, Aguiar RC. Targeting of SMAD5 links microRNA-155 to the TGF-beta pathway and lymphomagenesis. Proc Natl Acad Sci U S A. 2010;107(7):3111-6.

20. Zhao Y, Cui G, Zhang N, Liu Z, Sun W, Peng Q. Lipopolysaccharide induces endothelial cell apoptosis via activation of $\mathrm{Na}(+) / \mathrm{H}(+)$ exchanger 1 and calpain-dependent degradation of Bcl-2. Biochem Biophys Res Commun. 2012:427(1):125-32.

21. Feng $D$, Ling $W H$, Duan RD. Lycopene suppresses LPS-induced $N O$ and $I L-6$ production by inhibiting the activation of ERK, p38MAPK, and NF-kappa B in macrophages. Inflamm Res. 2010;59(2):115-21.

22. Peng QS, Long CL, Malhotra S, Humphrey MB. A Physical Interaction Between the Adaptor Proteins DOK3 and DAP12 Is Required to Inhibit Lipopolysaccharide Signaling in Macrophages. Sci Signal. 2013;6:289.

23. Cui G, Wei P, Zhao Y, Guan Z, Yang L, Sun W, Wang S, Peng Q Brucella infection inhibits macrophages apoptosis via Nedd4-dependent degradation of calpain2. Vet Microbiol. 2014;174(1-2):195-205.

24. Boone DL, Turer EE, Lee EG, Ahmad RC, Wheeler MT, Tsui C, Hurley P, Chien M, Chai S, Hitotsumatsu $\mathrm{O}$, et al. The ubiquitin-modifying enzyme A20 is required for termination of Toll-like receptor responses. Nat Immunol. 2004; 5(10):1052-60.

25. Ackermann MR, Cheville NF, Deyoe BL. Bovine ileal dome lymphoepithelial cells: endocytosis and transport of Brucella abortus strain 19. Vet Pathol. 1988;25(1):28-35.

26. Jiang $X$, Baldwin CL. Effects of cytokines on intracellular growth of Brucella abortus. Infect Immun. 1993;61(1):124-34. 
27. Dornand J, Gross A, Lafont V, Liautard J, Oliaro J, Liautard JP. The innate immune response against Brucella in humans. Vet Microbiol. 2002;90(1-4):383-94.

28. Gross A, Bertholet S, Mauel J, Dornand J. Impairment of Brucella growth in human macrophagic cells that produce nitric oxide. Microb Pathog. 2004; 36(2):75-82.

29. Kawai T, Akira S. The role of pattern-recognition receptors in innate immunity: update on Toll-like receptors. Nat Immunol. 2010;1 1(5):373-84.

30. von Bargen K, Gorvel JP, Salcedo SP. Internal affairs: investigating the Brucella intracellular lifestyle. Fems Microbiology Reviews. 2012;36(3):533-62.

31. Karin M. Nuclear factor-kappaB in cancer development and progression. Nature. 2006;441(7092):431-6.

Submit your next manuscript to BioMed Central and we will help you at every step:

- We accept pre-submission inquiries

- Our selector tool helps you to find the most relevant journal

- We provide round the clock customer support

- Convenient online submission

- Thorough peer review

- Inclusion in PubMed and all major indexing services

- Maximum visibility for your research

Submit your manuscript at www.biomedcentral.com/submit
Biomed Central 